\title{
Coarse-graining the dynamics of coupled oscillators
}

\author{
Sung Joon Moon ${ }^{1}$, R. Ghanem ${ }^{2}$, and I. G. Kevrekidis ${ }^{1 *}$ \\ ${ }^{1}$ Department of Chemical Engineering $\&$ Program in Applied and Computational Mathematics (PACM), \\ Princeton University, Princeton, NJ 08544, USA \\ ${ }^{2}$ Department of Civil Engineering, University of Southern California, Los Angeles, CA 90089, USA
}

(Dated: November 19, 2018)

\begin{abstract}
We present an equation-free computational approach to the study of the coarse-grained dynamics of finite assemblies of non-identical coupled oscillators at and near full synchronization. We use coarse-grained observables which account for the (rapidly developing) correlations between phase angles and oscillator natural frequencies. Exploiting short bursts of appropriately initialized detailed simulations, we circumvent the derivation of closures for the long-term dynamics of the assembly statistics.

PACS numbers: 05.45.Xt,05.10.-a,02.70.Dh,87.10.+e
\end{abstract}

Since Winfree's pioneering work in 1960's [1], coupled oscillator models have been investigated extensively. Some exact results on the collective dynamics for an infinite number of coupled oscillators (the so-called continuum-limit) 2, 3, 44 have shed light on synchronization phenomena in biological $1,[2,5,6,6$, 8], chemical [9, 10], and physical systems [11, 12]. However, even in this ideal limit, some basic questions including global, quantitative stability of asymptotic states, still remain open 13, 14, 15, 16]. Many real-world systems consist of a large, finite number of non-identical entities, where statistical techniques for the continuum-limit are not directly applicable. Exploring and understanding the dynamics of such finite oscillator assemblies is an important topic (e.g., see Ref. [17]).

We present a computer-assisted approach to modeling the coarse-grained dynamics of such large, finite oscillator assemblies at and near full synchronization. The premise is that there exist a small number of coarse-grained variables (observables) adequately describing the long-term dynamics, and that a closed evolution equation for these observables exists, but is not explicitly available. To account for oscillator variability within the assembly, we treat both the variable oscillator properties (here, natural frequencies $\omega$ ) and the oscillator states (here, phase angles $\theta$ ) as random variables. Recognizing a quick development of correlations between $\omega$ and $\theta$, we express the latter as a polynomial expansion of the former (borrowing Wiener polynomial chaos (PC) tools [18]); the PC expansion coefficients are our coarse observables.

Availability of the governing equations for the variables of interest is a prerequisite to modeling and computation. We circumvent this step using the recently-developed equation-free (EF) framework for complex, multiscale systems modeling [19, 20, 21]. In this framework we can perform system-level computational tasks without explicit knowledge of the coarse-grained equations; these unavailable equations are solved by designing, performing and processing the results of short bursts of appropriately initialized detailed (fine-scale, microscopic) simulations.
We consider a paradigmatic model of coupled oscillators, the Kuramoto model, consisting of a population of $N$ all-to-all, phase-coupled limit-cycle oscillators with i.i.d. $\omega$ with distribution function $g(\omega)$. This model has been extensively studied because of its simplicity and certain mathematical tractability, yet it is not merely a toy model. It appears as a normal form for general systems of coupled oscillators (e.g. Refs. 10, 11]).

We choose a Gaussian with standard deviation $\sigma_{\omega}=$ 0.1 for $g(\omega)$; however, our approach is not limited to this particular choice, nor to the Kuramoto model. Due to rotational symmetry, the mean frequency $\Omega=\sum_{i} \omega_{i} / N$ can be set to 0 without loss of generality. The governing equation for the phase angle of the $i$ th oscillator $\theta_{i}$ is

$$
\frac{d \theta_{i}}{d t}=\omega_{i}+\frac{K}{N} \sum_{j=1}^{N} \sin \left(\theta_{j}-\theta_{i}\right), \quad 1 \leq i \leq N,
$$

where $K \geq 0$ is the coupling strength. Spontaneous synchronization (phase-locking) occurs at sufficiently large $K$. As $K$ decreases across a critical value $K_{t p}$, more and more oscillators desynchronize until they all essentially evolve with their own frequencies below another critical value $K_{c}[3,13,15]$. Kuramoto [3] introduced a complex order parameter $r e^{i \psi}=\frac{1}{N} \sum_{j=1}^{N} e^{i \theta_{j}}$ to describe the longtime states; the effective radius $r(t)$ measures the phase coherence; see also Ref. 22] for an order function. The asymptotic value of $r(t \rightarrow \infty)$ in the continuum-limit $(N \rightarrow \infty)$ exhibits a temporal analog of phase transition at $K_{c}[3]$.

The order parameter $r$ conveniently represents statistical behaviors around the critical point $K=K_{c}$; however, $r$ does not uniquely specify the microscopic state, and it may not adequately describe transient dynamics. The statistical moments of the phase angle distribution function $\mathcal{M}_{n} \equiv \frac{1}{N} \sum_{j=1}^{N}\left(\theta_{j}-\frac{1}{N} \sum_{i=1}^{N} \theta_{i}\right)^{n}$, where $n$ is a positive integer, are a "natural" first choice of coarsegrained observables (in a kinetic theory-like description). Due to the symmetry, we consider only even-order moments, and test whether a closure in terms of $\mathcal{M}_{2}$ and 


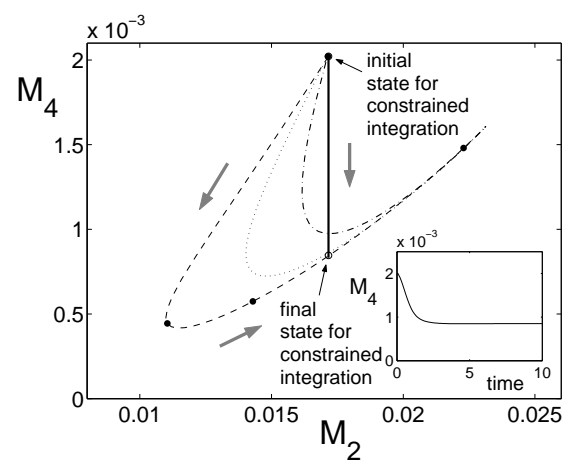

FIG. 1: Three coarsely identical (same $\mathcal{M}_{2}$ and $\mathcal{M}_{4}$ ) but microscopically different initializations (dashed, dotted and dot-dashed lines; see text) evolve along different trajectories, to a slow manifold and, ultimately, the same synchronized state $\left(N=300 ; K=0.7>K_{t p}\right)$. Constraining the evolution to $\mathcal{M}_{2}=0.017$ (solid line) guides the trajectory directly to this slow manifold; the inset shows $\mathcal{M}_{4}$ becoming slaved to $\mathcal{M}_{2}$ during this constrained evolution by $t \approx 2.0$.

$\mathcal{M}_{4}$ is likely for $K \geq K_{t p}$. We prepare several distinct initial phase angle distributions with identical coarsegrained values $\left(\mathcal{M}_{2}=0.017\right.$ and $\left.\mathcal{M}_{4}=0.0020\right)$; these phase angles are randomly assigned to oscillators. The phase portrait in Fig. 1] shows direct simulation [using Eq. (11) for three of these initial assemblies; the trajectories are clearly distinct, suggesting that the dynamics cannot close simply on these two observables. Including higher order moments (such as $\mathcal{M}_{6}$ ) as observables does not remedy the situation. It is also clear, however, that the long-term dynamics lie on a low-dimensional manifold (ultimately a one-dimensional one) towards which all trajectories are eventually attracted.

The dynamical differences among the three cases arise from the microscopic differences of the (macroscopically identical) initial conditions; this is best seen in the $\omega-\theta$ plane (Fig. 2). Correlations between $\theta$ and $\omega$ develop (the initial "cloud" in the $\omega-\theta$ plane quickly evolves to a "curve"), as all transients initially approach the slow manifold: The oscillators "sort themselves out". These correlations were not accounted for when we assigned angles randomly to oscillators in the assembly.

We now include a "remedial initialization" step, evolving the dynamics by constraining them on prescribed values of the moments, as a system of differential algebraic equations (DAEs) using Lagrange multipliers. The solid line in Fig. 1 shows this preparatory step with a constraint on $\mathcal{M}_{2}$ only; constrained evolution brings the assembly down to the right point on the slow manifold, and the same "sorting" develops as in the aforementioned freely-evolving cases. Phase angle statistics alone do not, therefore, constitute good observables 23]; $\omega-\theta$ correlations should be accounted for in the coarse description.

Motivated by this observation, we explore the long-

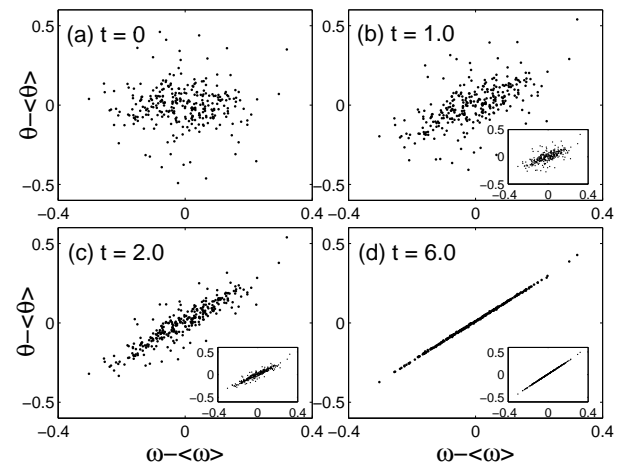

FIG. 2: Time snapshots in the $\omega-\theta$ plane for free evolution (main panels; dashed line in Fig. 1) and for constrained evolution (insets; solid line in Fig. 1) respectively. Each dot represents an oscillator, and (a) to (d) are snapshots at $\mathrm{t}=0$, 1.0, 2.0, and 6.0, respectively, marked by filled circles in Fig.1 Strong correlations develop during the initial transient stages ("oscillator sorting").

term dynamics with a different set of observables, treating both $\theta$ and $\omega$ as random variables. The former is expanded in Hermite polynomials of the latter, Gaussian random variable; Wiener polynomial chaos is the appropriate choice for Gaussian distributions. Generalized polynomial chaos (gPC) 24 would be invoked for different frequency distributions (e.g. we also successfully used Legendre polynomial expansions for uniform $g(\omega)$ ). For convenience, we introduce the normalized random variable $\xi \equiv \omega / \sigma_{\omega}$ :

$$
\theta(\omega, t)=\sum_{i=0}^{p} \alpha_{i}(t) H_{i}(\xi)=\sum_{i=0}^{p} \frac{\left\langle\theta, H_{i}\right\rangle}{\left\langle H_{i}, H_{i}\right\rangle} H_{i}(\xi)
$$

where $p$ is the highest order retained in the truncated series, $\langle\cdot, \cdot\rangle$ denotes the inner product with respect to the Gaussian measure, and $H_{i}$ is the $i$ th Hermite polynomial $\left[H_{0}(x)=1, H_{1}(x)=x, H_{2}(x)=x^{2}-1, H_{3}(x)=\right.$ $\left.x^{3}-3 x, \cdots\right]$. Only odd-order $\alpha_{i}$ 's are considered, due to symmetry. We will see that here the first two nonvanishing coefficients $\alpha_{1}$ and $\alpha_{3}$ provide an adequate representation. Given a particular detailed realization of the oscillator state, its PC coefficients $\alpha_{i}$ 's are estimated through a least squares fitting algorithm, interpreting $\theta$ as an empirical function $f(\xi) \equiv \alpha_{1} \xi+\alpha_{3}\left(\xi^{3}-3 \xi\right)$ and minimizing the residual $R^{2} \equiv \sum_{j}\left[\theta_{j}-f\left(\xi_{j} ; \alpha_{1}, \alpha_{3}\right)\right]^{2}$. This procedure corresponds to the restriction (fine to coarse) step in the EF framework, described below.

In the EF approach, appropriately initialized short bursts of detailed, fine-scale simulation are used to estimate quantities pertaining to the evolution of the coarsegrained variables (observables). Lacking an explicit coarse-grained model in terms of the first few PC coefficients, we estimate the quantities necessary for scientific computation with it (time derivatives, action of Jaco- 

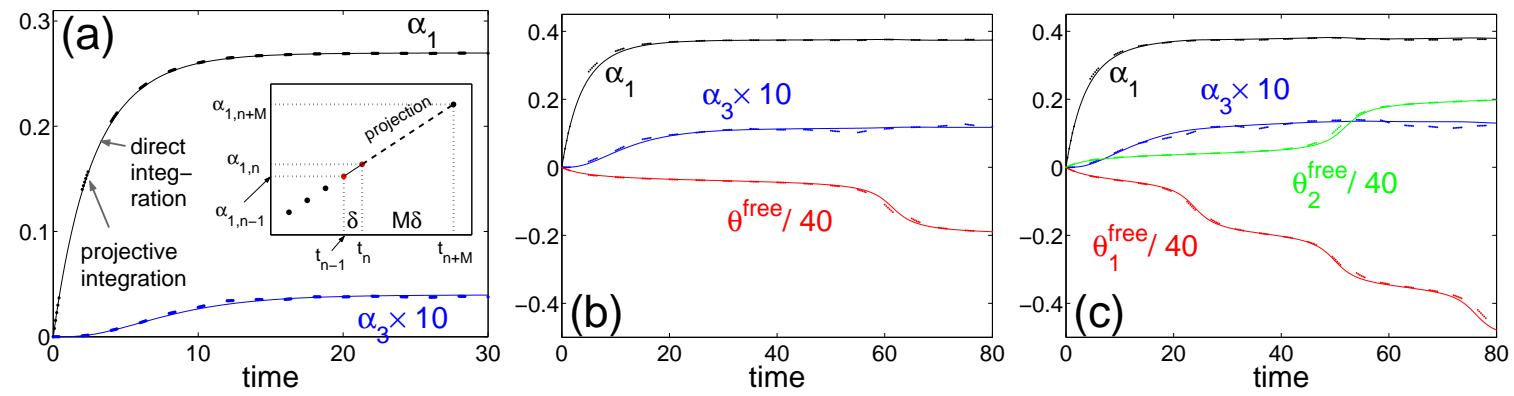

FIG. 3: (color online) Coarse projective integration (dots) and detailed coupled oscillator dynamics (lines); $N=300$. (a) Two PC coefficients $(K=0.4$; full synchronization). (b) Two PC coefficients and a single "free" oscillator $(K=0.31)$. (c) Two PC coefficients and two "free" oscillators $(K=0.31)$. Natural frequencies are newly drawn from $g(\omega)$ at each lifting step (see text). Inset in (a): Schematic of a projective integration step: The last part (last two dots, at $t_{n-1}$ and $t_{n}$ ) of a short burst of direct integration (five dots) is used to estimate the local time derivative (solid line). PC values at a future time $t=t_{n+M}$ are "projected" through forward Euler, i.e., $\left.\alpha_{1}\right|_{t=t_{n+M}}=\left.\alpha_{1}\right|_{t=t_{n}}+\frac{\alpha_{1, n}-\alpha_{1, n-1}}{t_{n}-t_{n-1}}\left(t_{n+M}-t_{n}\right)$.

bians, residuals) through on demand numerical experimentation with the detailed, fine-scale model [Eq. (1)].

The general procedure consists of $(i)$ identifying good observables that sufficiently describe the coarse-grained dynamical state (here, a few $\alpha_{i}$ 's), (ii) constructing a lifting operator, mapping the coarse description to one (or more, for variance reduction purposes) consistent finescale realization(s) [randomly drawing $\omega$ from $g(\omega)$ and assigning $\theta$, using Eq. (2) and given $\alpha_{i}$ values], (iii) evolving the lifted, fine-scale initial conditions for certain time horizon, ( $i v)$ restricting the resulting fine-scale description to the coarse observables [finding the $\mathrm{PC}$ coefficients of the final state], and $(v)$ repeating the procedure as necessary to perform specific scientific computation steps. This is a general approach that has been combined with various fine-scale models [19, 25]; see Refs. [20, 21].

We first demonstrate coarse projective integration [26]. Each group of five dots in Fig. [3] represents the time horizon during which the detailed equations are integrated to enable the projective step; the local time derivatives of the observables are estimated here simply from the last two dots in each group. Coarse variable values at a projected, future time are estimated using these derivatives and (for projective forward Euler) linear extrapolation in time [see the inset in Fig. 3 (a)]. After the projection step we lift the coarse variables to consistent fine-scale realizations, and use these as the initial condition for another short burst of direct detailed integration [steps (ii) and (iii) above]. Depending on the relative lengths of the projection step $(M \delta)$ and the short run required to estimate the coarse time derivatives $(n \delta)$, this procedure may significantly accelerate the computational evolution of the oscillator statistics; the cost of the lifting step (here negligible) must also be considered. At each lifting step, $\omega$ was newly drawn from $g(\omega)$, and the full integration (lines) and projective integration (dots) agree on the level of fluctuation among realizations. The PC coefficients display smooth behavior, nearly independently of particular random draws; for the same random draw at every step, results would be even better. Projective integration in Fig. 3] (a) reduced the computational effort in our illustrative direct integration by a factor of four. The numerical analysis of projective integration (stability, accuracy, stepsize selection and estimation issues) is a topic of current research (see e.g., Refs. [20, 26, 27]); here we simply demonstrated the procedure and its potential.

Slightly below the transition value $K_{t p}$, where only few oscillators become desynchronized, we consider the system as a combination of synchronized "bulk" and a few "free" oscillators. Good coarse-grained observables then are a few PC coefficients for the "bulk" synchronized oscillators and the phase angle(s) of the (few) desynchronized one(s). The EF approach can be directly "wrapped around" this alternative representation. Both for the one free and two free oscillator cases, projective integrations on the new observables successfully track (and accelerate) direct detailed simulations [Figs. 3] (b) and (c)]. These "good observables" are suggested by direct inspection and common sense; for more complicated, highdimensional problems, good state parameterizations require modern data mining algorithms. Diffusion maps on graphs constructed by the data [29] are a promising tool for detecting good "reduction coordinates" (observables) on which to base EF computations.

Direct, long-time simulation is often inefficient in computing long-time (stationary) states. Numerical bifurcation algorithms, more appropriate for stability and parametric analysis, can be implemented in an equation-free framework: The residual and the action of the unavailable Jacobian are numerically estimated through short bursts of appropriately initialized detailed simulations. Starting from a coarse-grained initial condition, we lift, and integrate the full model for time $\Delta T$. We then restrict to the observables of the final state $\Phi_{\Delta T}$; this is the 


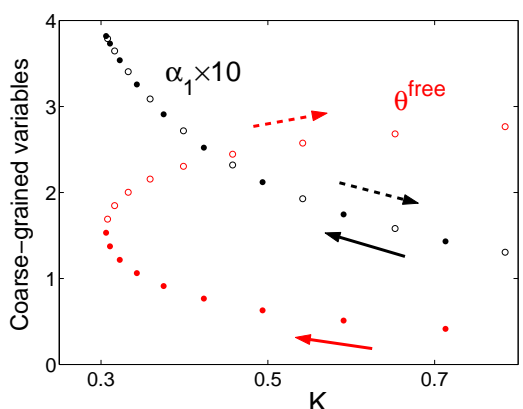

FIG. 4: (color online) Coarse bifurcation diagram for the full synchronization regime $\left(K \geq K_{t p}\right)$, obtained through coarse Newton-GMRES method and pseudo arc-length continuation $(N=300)$. The same variables as in Fig. [3 (b) are used; the phase angle of the single "free" oscillator $\left(\theta^{\text {free }}\right)$ is an extra observable (its natural frequency is positive in this case). The PC coefficients, obtained by discounting the "free" oscillator, exhibit nearly the same values both for the stable (filled circles) and the unstable (open circles) branch (only $\alpha_{1}$ is shown here). Only $\theta^{\text {free }}$ shows significant variation along the two branches. Arrows are included to guide the eye.

coarse time-stepper. We now solve for the fixed point satisfying $\Phi_{\Delta T}\left(\begin{array}{c}\boldsymbol{\alpha} \\ \boldsymbol{\theta}^{\text {free }}\end{array}\right)-\left(\begin{array}{c}\boldsymbol{\alpha} \\ \boldsymbol{\theta}^{\text {free }}\end{array}\right)=0$, using the coarse Newton-GMRES [28], a matrix-free iterative method (together with the pseudo arc-length continuation); additional coarse observables $\boldsymbol{\theta}^{\text {free }}$ are appended when necessary. We construct bifurcation diagrams like the one in (Fig. 4) with respect to the parameter $K$, showing a turning point (actually, a "sniper") bifurcation at $K=K_{t p}$. A single oscillator (whose phase angle $\theta^{\text {free }}$ is treated as a separate coarse observable) becoming "free" from the synchronized "bulk" at that point. For sufficiently large $K$ values (when $r \approx 1$ ) analytical estimates of certain elements of the shape of the $\omega-\theta$ correlation become possible (e.g., from Eq. (1) one can obtain, at steady state, $\alpha_{1} \approx \sigma_{\omega} / K$, in reasonable agreement with our steady state computations at $K>\sim 0.5)$.

In summary, the EF multiscale approach was successfully used for coarse-grained dynamic computations of finite assemblies of non-identical coupled oscillators; the derivation of explicit closures at- and close to the synchronization regime was circumvented. Initial transient "sorting" of the oscillators, establishing correlations between natural frequencies and phase angles, suggested Wiener PC coefficients as the appropriate coarse observables. If the problem dynamics can be coarse-grained, traditional numerical analysis algorithms can be used as protocols for the "intelligent" design of short bursts of computational experiments with the detailed, fine-scale model. The approach can be directly generalized to analyze the simulation and modeling of more complicated oscillator dynamics.

This work was supported by DARPA and by the Na- tional Science Foundation.

* yannis@arnold.princeton.edu

[1] A. T. Winfree, J. Theor. Biol. 16, 15 (1967).

[2] Y. Kuramoto, Chemical Oscillations, Waves, and Turbulence, Springer-Verlag (1984).

[3] Y. Kuramoto, in International Symposium on Mathematical Problems in Theoretical Physics, Edited by $\mathrm{H}$. Arakai, Lecture Notes in Physics, Vol. 39, (Springer, New York, 1975), p. 420.

[4] J. T. Ariaratnam and S. H. Strogatz, Phys. Rev. Lett. 86, 4278 (2001).

[5] C. M. Gray, P. Konig, A. K. Engel, and W. Singer, Nature 338, 334 (1989).

[6] J. Buck, Quart. Rev. Biol. 63, 265 (1988).

[7] T. J. Walker, Science 166, 891 (1969).

[8] Z. Néda, E. Ravasz, Y. Brechet, T. Vicsek, and A. L. Barabási, Nature 403, 849 (2000).

[9] G. Ertl, Science 254, 1750 (1991).

[10] I. Z. Kiss, Y. Zhai, and J. L. Hudson, Science 296, 1676 (2002).

[11] K. Wiesenfeld, P. Colet, and S. H. Strogatz, Phys. Rev. Lett. 76, 404 (1996).

[12] R. A. Oliva and S. H. Strogatz, Int. J. Bifur. Chaos 11, 2359 (2001).

[13] S. Strogatz, Physica D 143, 1 (2000).

[14] A. Pikovsky, M. Rosenblum, and J. Kurths, Synchronization, Cambridge University Press, Cambridge (2001)

[15] S. C. Manrubia, A. S. Mikhailov, and D. H. Zanette, Emergence of Dynamical Order, World Scientific, Singapore (2004).

[16] J. A. Acebrón, L. L. Bonilla, C. J. Pérez Vicente, F. Ritort, and R. Spigler, Rev. Mod. Phys. 77, 137 (2005).

[17] N. J. Balmforth and S. Sassi, Physica D 143, 21 (2000).

[18] R. Ghanem and P. Spanos, Stochastic Finite Elements: A Spectral Approach, Springer-Verlag, New York (1991).

[19] C. Theodoropoulos, Y. H. Qian, and I. G. Kevrekidis, Proc. Natl. Acad. Sci. USA 97, 9840 (2000).

[20] I. G. Kevrekidis et al., Comm. Math. Sciences 1 (4), 715 (2003); e-print physics/0209043

[21] I. G. Kevrekidis, C. W. Gear, and G. Hummer, AIChE J. 50, 1346 (2004).

[22] H. Daido, Phys. Rev. Lett. 73, 760 (1994).

[23] S. J. Moon and I. G. Kevrekidis, Int. J. Bifur. Chaos, in press; e-print nlin.AO/0502016

[24] D. Xiu and G. Em Karniadakis, SIAM J. Sci. Comput. 24, 619 (2002).

[25] C. W. Gear, I. G. Kevrekidis, and C. Theodoropoulos, Comp. Chem. Engng. 26, 941 (2002); A. G. Makeev et al., J. Chem. Phys. 117, 8229 (2002); C. Siettos, M. D. Graham, and I. G. Kevrekidis, ibid. 118, 10149 (2003); G. Hummer and I. G. Kevrekidis, ibid. 118, 10762 (2003).

[26] C. W. Gear and I. G. Kevrekidis, SIAM J. Sci. Comput. 24, 1091 (2003).

[27] R. Rico-Martinez, C. W. Gear, and I. G. Kevrekidis, J. Comp. Phys. 196, 474 (2004).

[28] C. T. Kelley, Iterative Methods for Linear and Nonlinear Equations, SIAM, Philadelphia (1995).

[29] B. Nadler, S. Lafon, R. C. Coifman, and I. G. Kevrekidis, Appl. Comp. Harm. Anal. in press; e-print 
math/0503445 